\title{
CARACTERÍSTICAS QUÍMICAS DE ABACAXI 'PÉROLA' APÓS TRATAMENTO COM CALOR E ARMAZENAGEM EM TRÊS TEMPERATURAS ${ }^{1}$
}

\author{
ROSILENE FERREIRA SOUTO², JOSÉ FERNANDO DURIGAN ${ }^{3}$, \\ LEANDRA OLIVEIRA SANTOS ${ }^{4}$, BIANCA SARZI DE SOUZA ${ }^{5}$, \\ JOÃO LUIZ PALMA MENEGUCCI ${ }^{6}$
}

RESUMO- Avaliou-se o efeito do tratamento térmico $\left(40^{\circ} \mathrm{C}\right.$ por 24 horas) e de diferentes temperaturas de armazenamento $\left(8^{\circ} \mathrm{C}, 14^{\circ} \mathrm{C}\right.$ e $25^{\circ} \mathrm{C}$, a $\left.90 \% \mathrm{UR}\right)$, na conservação pós-colheita de abacaxis 'Pérola', colhidos no ponto de maturação “pintado”. As avaliações foram realizadas no início (0 dia), visando à caracterização inicial dos frutos, e após 1; 5; 9; 13 e 17 dias, quando os mantidos sob refrigeração foram transferidos para condição ambiente $\left(25^{\circ} \mathrm{C}, 75-80 \%\right.$ UR), e avaliados aos 21; 25 e 29 dias. O delineamento experimental utilizado foi o inteiramente casualizado, em esquema fatorial ( $2 \times 3 \times 9)$, tendo-se os frutos tratados termicamente ou não, o armazenamento a $25^{\circ} \mathrm{C}, 14^{\circ} \mathrm{C}$ e $8^{\circ} \mathrm{C}$ e nove épocas de avaliação. Os frutos foram avaliados quanto à ocorrência de podridões e de escurecimento interno, aparência e coloração da polpa, teores de sólidos solúveis (SS), acidez titulável (AT), açúcares solúveis totais e redutores e ácido ascórbico, além da relação SS/AT. Os resultados indicam que a coloração da polpa se tornou mais amarela durante o período refrigerado, enquanto os valores da AT aumentaram. Neste período, a relação SS/AT reduziu-se, mas aumentou com a transferência dos frutos para o ambiente, enquanto os teores de açúcares solúveis totais e redutores diminuíram, e estabilizaram-se. Os teores de ácido ascórbico mantiveram-se sem diferenças significativas, mas com tendência de aumento. Os frutos mantidos sob refrigeração apresentaram sintomas de injúria pelo frio, que apareceram em 8 dias, após serem levados ao ambiente, e com maior intensidade nos tratados termicamente.

Termos para indexação: Ananas comosus, armazenamento, escurecimento interno.

\section{PINEAPPLE 'PÉROLA' CHEMICAL CHARACTERISTICS AFTER ROT TREATMENT AND STORAGE IN THREE TEMPERATURE}

\begin{abstract}
The effects of heating the fruits at $40^{\circ} \mathrm{C}$ for 24 hours and different storage temperatures $\left(8^{\circ} \mathrm{C}\right.$, $14^{\circ} \mathrm{C}$ and $25^{\circ} \mathrm{C}$, at $\left.90 \% \mathrm{RH}\right)$ to postharvest conservation of 'Pérola' pineapple fruit, harvested on "pintado" maturation stage. The evaluations were carried at the beginning (day 0), for initial characterization of the fruit and after 1, 5, 9, 13, and 17 days, with the fruit kept under refrigeration were transferred to environmental condition at $25^{\circ} \mathrm{C}$ and $75-80 \% \mathrm{RH}$ and evaluated at the 21, 25, 29 days. It was used an entirely randomized experimental design, in a factorial scheme of $2 \times 3 \times 9$, with fruit thermally treated or non-treated fruits, storage at $25^{\circ} \mathrm{C}, 14^{\circ} \mathrm{C}$, and $8^{\circ} \mathrm{C}$ and 9 different evaluation periods. The fruit were evaluated for rottenness and pulp darkening incidence, pulp color and appearance, soluble solids (SS), titratable acidity (AT), soluble and reduced sugars and ascorbic acid content, besides SS/AT ratio. The results indicate that the pulp color became yellower during the refrigerated period, while the titratable acidity increased. During this period, the SS/AT rate lowered, but increased when the fruit were transferred to the environment, while the soluble and reduced sugars content decreased initially, before stabilizing. The ascorbic acid content had no significant variations, but showed a tendency of increasing. The fruit kept under environmental conditions did not show signs of chilling injury that were observed in the fruits after 8 days at refrigeration, after being taken out to the environmental conditions, with greater intensity in those thermally treated fruits.

Index terms: Ananas comosus, storage, internal darkening.

\footnotetext{
${ }^{1}$ (Trabalho 004-09). Recebido em: 05-01-2009. Aceito para publicação em: 08-01-2010.

${ }^{2}$ Eng $^{\mathrm{a}}$. Agr ${ }^{\mathrm{a}}$., Fiscal Federal, DFA-GO, Goiânia-GO. Praça Cívica, 100. Centro, Goiânia-GO, e-mail: rosilenef@agricultura.gov.br

${ }^{3}$ Eng $^{\circ}$. Agr ${ }^{\circ}$., Prof. Titular do Departamento de Tecnologia da FCAV/UNESP, Jaboticabal. Via de acesso Prof. Paulo Donato Castellane, s/n, 14.884-900, Jaboticabal-SP, e-mail: jfduri@fcav.unesp.br

${ }^{4}$ Eng $^{\mathrm{a}}$. Agr ${ }^{\mathrm{a}}$., Doutoranda em produção Vegetal da FCAV/UNESP, Jaboticabal, Via de Acesso Prof. Paulo Donato Castellane, s/n, 14884-9000, Jaboticabal-SP, e-mail: leandraoli@yahoo.com.br

${ }^{5}$ Eng $^{\text {a }}$. Agra ${ }^{\mathrm{a}}$, D.Sc. Assistente Agropecuário da Coordenadoria de Defesa Agropecuária - IDA Casa Branca, Praça Honório de Sillos nº 13 - Centro, 13700-000, Casa Branca - SP, e-mail: bianca.souza@cda.sp.gov.br

${ }^{6}$ Eng $^{\circ}$. Agr ${ }^{\circ}$., D.Sc. Pesquisador Embrapa Mandioca e Fruticultura, Escritório de Negócios de Goiânia. Rod. BR153. km 04, e-mail: meneguci@cnpmf.embrapa.br
} 


\section{INTRODUÇÃO}

A refrigeração não é fator suficiente para prolongar a vida útil pós-colheita e evitar mudanças indesejáveis na qualidade de abacaxis, apesar de ser muito utilizada durante o transporte, pois pode provocar o distúrbio fisiológico conhecido como escurecimento interno (Bleinroth, 1987). Souto et al. (2004) estudaram a associação da refrigeração e atmosfera modificada na conservação de abacaxis 'Pérola' e observaram que o escurecimento interno se manifestou após a transferência dos frutos da condição refrigerada $\left(8^{\circ} \mathrm{C}\right)$ para o ambiente $\left(25^{\circ} \mathrm{C}\right.$, 75-80\% UR), em 4 dias.

Um fator determinante no uso de diferentes temperaturas de estocagem é o estádio de maturação dos frutos. Garcia et al. (1996) recomendam para frutos maduros e meio-maduros, $7-10^{\circ} \mathrm{C}$, e para os verde-maduros, $10-13^{\circ} \mathrm{C}$, enquanto para Carvalho (1999), a temperatura ideal é $8^{\circ} \mathrm{C}$. Chitarra \& Chitarra (2005) recomendam, para o armazenamento refrigerado de abacaxis, temperaturas que variam de $7^{\circ} \mathrm{C}$ a $13^{\circ} \mathrm{C}$, com umidade relativa de $85-90 \%$.

Na conservação pós-colheita do abacaxi, outras técnicas têm sido associadas à refrigeração, como o tratamento térmico anterior ao armazenamento refrigerado para se evitar a ocorrência do escurecimento interno da polpa, devido à inativação das enzimas responsáveis (Gonçalves, 1998). Akamine (1976) indicou a exposição dos frutos a $38^{\circ} \mathrm{C}$ por 24 horas, antes de serem armazenados ou transportados a $10-12^{\circ} \mathrm{C}$.

Este trabalho teve por objetivo determinar o efeito do tratamento térmico e de diferentes temperaturas de armazenamento, na conservação póscolheita de abacaxis 'Pérola', colhidos “pintados”.

\section{MATERIAL E MÉTODOS}

A colheita dos frutos foi realizada manualmente, em novembro de 2001, no município de Frutal-MG, no estádio de maturação "pintado”, que se caracteriza pelos frutilhos com centro amarelado. O peso médio dos frutos era de 1.470,5 g, diâmetro de 11,4 cm e comprimento de 37,2 cm ou Classe 2, de acordo com o Programa Brasileiro para a Melhoria dos Padrões Comerciais e Embalagens de Hortigranjeiros (CEAGESP, 2000). O comprimento e o peso da coroa eram $18,8 \mathrm{~cm}$ e 104,7 g, respectivamente. Os frutos foram cuidadosamente transportados para o laboratório de Tecnologia dos Produtos Agrícolas da FCAV, UNESP, onde foram novamente selecionados, lavados com detergente neutro, imersos em emulsão do fungicida Sportak $450 \mathrm{EC}^{\circledR}$ (procloraz 45\%) a
$1.000 \mathrm{mg} \mathrm{L}^{-1}$, a $10^{\circ} \mathrm{C}$, por 20 minutos

Os frutos foram divididos aleatoriamente em 2 grupos, sendo um mantido em estufa $\left(40^{\circ} \mathrm{C}\right.$ por 24 horas) e o outro sob condição de ambiente $\left(25^{\circ} \mathrm{C}\right.$ e $75-80 \%$ UR). Cada grupo foi então dividido em três lotes iguais, que receberam os seguintes tratamentos: testemunha, frutos que permaneceram armazenados sob condição ambiente $\left(25^{\circ} \mathrm{C}\right.$; 75-80\% UR), por 17 dias; e frutos armazenados a $14^{\circ} \mathrm{C}(90 \%$ UR), ou a $8^{\circ} \mathrm{C}$ (90\% UR), por 17 dias, quando foram transferidos para a condição ambiente, onde foram mantidos por até 29 dias. Os frutos foram avaliados inicialmente, recém-colhidos (dia 0 ) ou depois de tratados termicamente (dia 1 ) e depois a cada 4 dias, ou seja, após 5; 9; 13; 17; 21; 25 e 29 dias. Este delineamento experimental permitiu ter como controle os frutos não tratados termicamente e os avaliados inicialmente.

Avaliaram-se os frutos quanto à coloração visual da polpa e ocorrência de escurecimento interno. Na polpa, também se determinaram os teores de sólidos solúveis, acidez titulável, ácido ascórbico, e de açúcares solúveis totais e redutores.

A coloração visual da polpa foi determinada conforme o indicado por Giacomelli (1982) e representada através de notas, em que: $1=$ branca ou quase branca; 2= amarela muito pálida; 3= amarela; 4= amarela intensa. Esta coloração também foi avaliada através de reflectometria, utilizando-se de um colorímetro Minolta CR-200b, que expressa este parâmetro, segundo o sistema proposto pela Commission Internacionale de L'Eclaraige (CIE), e permite expressar a coloração em luminosidade (L), cromaticidade e ângulo hue ou de cor (Wolf et al., 1997).

O índice de escurecimento interno (EI) foi determinado, em cada fruto, pela expressão (EI), ou seja: EI= \% de área afetada $\mathrm{x}$ intensidade do EI. A porcentagem da área afetada era calculada pela região entre as áreas escurecida e total, determinadas com planímetro digital no fruto cortado longitudinalmente ao meio. A intensidade do escurecimento interno foi determinada visualmente, usando-se uma escala de notas, em que: $0=$ ausência de escurecimento; $1=$ marrom-claro; 2= marrom-médio; 3= marrom-escuro (Souto et al., 2004).

Os teores de sólidos solúveis (\%) foram determinados por refratometria; os de acidez titulável por titulação com solução de $\mathrm{NaOH}$ padronizada e expressos em gramas de ácido cítrico por $100 \mathrm{~mL}$ de suco (AOAC, 1997). O teor de ácido ascórbico foi determinado pelo método de Tillman (Strohecker \& Henning, 1967); o de açúcares solúveis totais pela técnica do fenolsulfúrico (Dubois et al., 1956); e o 
de açúcares redutores conforme a técnica do ácido 3,5 dinitrossalicílico (Miller, 1959).

O delineamento experimental utilizado foi o inteiramente casualizado, em esquema fatorial (2x3x9), com os fatores, tipo de tratamento (térmico e ambiente), temperatura de armazenamento (ambiente, $14^{\circ} \mathrm{C}$ e $8^{\circ} \mathrm{C}$ ) e épocas de avaliação, com três repetições e unidade experimental composta por dois frutos. As comparações entre as médias dos tratamentos foram realizadas utilizando-se do teste de Tukey, ao nível de $5 \%$ de probabilidade, para as variáveis independentes, tratamento e temperatura de armazenamento.

\section{RESULTADOS E DISCUSSÃO}

O escurecimento interno (EI), manifestado após a transferência dos frutos armazenados a baixas temperaturas para condições de ambiente $\left(25^{\circ} \mathrm{C}\right.$ e $75-80 \%$ UR), foi detectado apenas após 8 dias, ou seja, no $25^{\circ}$ dia após a colheita. Nesta fase, somente os frutos tratados termicamente e que foram mantidos a $8^{\circ} \mathrm{C}$ e os não tratados, apresentaram este sintoma de injúria por chilling (Figura 1). Após 29 dias de armazenamento, os frutos de todos os tratamentos apresentaram sintomas de escurecimento interno e com maior intensidade que no $25^{\circ}$ dia, porém com redução considerável conseguida com o tratamento térmico e o armazenamento a $14^{\circ} \mathrm{C}$. Akamine (1976) também observou que o escurecimento interno da polpa do abacaxi pode ser minimizado pela exposição dos frutos a $38^{\circ} \mathrm{C}$, por 24 horas, antes de serem armazenados ou transportados a $10-12^{\circ} \mathrm{C}$. Tang-Yolin et al. (1997) trataram frutos da 'Smooth Cayenne' a $40^{\circ} \mathrm{C}$, por 24 horas, antes de armazená-los a $5^{\circ} \mathrm{C}, 7^{\circ} \mathrm{C}$ e $12^{\circ} \mathrm{C}$, por 4 semanas, e também observaram redução ou atraso no aparecimento do escurecimento interno. Selvarajah \& Herath (1997) estudaram o efeito do tratamento térmico, $32^{\circ} \mathrm{C} / 24$ horas, em frutos da cv. Mauritius, armazenados a $8^{\circ} \mathrm{C}$, durante 3 semanas, e observaram a ocorrência de escurecimento interno ao nível de $10 \%$.

Durante o período de armazenamento, a coloração visual da polpa tornou-se amarelo-pálida nos frutos mantidos a $25^{\circ} \mathrm{C}, 14^{\circ} \mathrm{C}$ e $8^{\circ} \mathrm{C}$, sem que o tratamento térmico inicial, a $40^{\circ} \mathrm{C}$, tivesse influência significativa. Esta coloração foi mantida após a transferência dos mesmos para a condição de ambiente (Tabela 1). A luminosidade, o ângulo hue e a cromaticidade da polpa não foram significativamente afetados pelos tratamentos (Tabelas 2; 3; 4), porém a evolução do ângulo de cor ( $\mathrm{h}^{\circ}$ ) e o aumento na cromaticidade indicaram amarelecimento, principalmente depois que os frutos foram levados para condições de ambiente (Tabelas 3 e 4), reafirmando o observado visualmente.

O teor de sólidos solúveis $\left(13,2^{\circ} \mathrm{Brix}\right)$ não foi significativamente alterado pelo tratamento térmico ou pelo tempo de armazenamento, pois evoluiu de 13,6 ${ }^{\circ}$ Brix para $13,3^{\circ}$ Brix nos 17 dias iniciais, e para $14,0^{\circ}$ Brix, após a transferência para o ambiente. Efeito semelhante também foi observado em abacaxis 'Smooth Cayenne' tratados termicamente a $40^{\circ} \mathrm{C}$ antes de serem armazenados sob refrigeração (Thé et al., 2001).

A acidez titulável (AT) aumentou com o período de armazenamento, passando de $0,49 \mathrm{~g}$ ácido cítrico $100 \mathrm{~g}^{-1}$ para $0,71 \mathrm{~g}$ ácido cítrico $100 \mathrm{~g}^{-1}$, em 17 dias, o que vem ao encontro do relatado por Silva(1997) e Botrel (1991). O tratamento térmico $\left(40^{\circ} \mathrm{C}\right)$ levou os frutos a apresentarem menor acidez que os não tratados, sem que as temperaturas de armazenamento levassem a alterações significativas (Tabela 5), atribuído a seu efeito redutor na atividade enzimática (Gonçalves et al., 2000; Thé et al., 2006).

Apesar de a relação entre os teores de SS e AT ter diminuído durante o período de armazenamento de 27,7 para até 19,3, com tendência à estabilidade após o quinto dia e depois da transferência dos frutos para o ambiente. Segundo Silva (1980),frutos com valores de SS/AT semelhantes a estes sabor adequado para consumo. Os frutos tratados termicamente apresentaram maior relação SS/AT durante o período de armazenamento, enquanto as temperaturas de armazenamento não mostraram influência nesta relação (Tabela 6), o que é consequência da evolução da acidez titulável. Gorgatti Neto et al. (1996) e Silva (1980) também observaram decréscimo nesta relação, tanto em frutos armazenados ao ambiente como a $12^{\circ} \mathrm{C}$, o que também foi relatado por Thé et al. (2001).

O conteúdo de açúcares solúveis totais na polpa diminuiu durante o período de armazenamento, sem que as temperaturas de armazenamento influenciassem nestes valores (Tabela 7), assim como não influenciaram nos teores de açúcares redutores (Tabela 8), que também apresentaram tendência de redução durante o armazenamento refrigerado. Estes teores mostraram tendência de aumento logo depois que os frutos foram transferidos à condição ambiente, antes de se estabilizarem. Thé et al. (2003), quando armazenaram frutos de abacaxi 'Smooth Cayenne’ sob refrigeração, após tratamento térmico inicial, também observaram redução nos teores de açúcares solúveis e redutores quando transferidos para condição de ambiente, indicando a ocorrência de hidrólise, o que foi atribuído à ação da invertase. O teor de ácido ascórbico aumentou durante 
o período de armazenamento inicial, ou até o $17^{\circ}$ dia, e não se modificou significativamente quando os frutos foram transferidos para a condição de ambiente (Tabela 9). Esta variabilidade no teor de ácido ascórbico também foi observada por Tang-Yolin et al. (1997), mas não indicado por Dull (1971), que não observou variação no teor deste constituinte durante o período de armazenamento.
O armazenamento de abacaxis 'Perola' sob diferentes condições, após serem submetidos a tratamento térmico, tornou a polpa dos mesmos amarelada, com teores de SS constantes, apesar da redução de açúcares solúveis totais e de açúcares redutores. A acidez titulável apresentou tendência de aumento, com consequente redução na relação SS/AT, enquanto nos teores de ácido ascórbico a tendência foi de aumento.

TABELA 1 - Aparência da polpa de abacaxis 'Pérola', colhidos no estádio de maturação pintado, tratados termicamente a $40^{\circ} \mathrm{C}$ ou não e armazenados por 17 dias, a $25^{\circ} \mathrm{C}$ (ambiente), $14^{\circ} \mathrm{C}$ e $8^{\circ} \mathrm{C}$, quando foram transferidos para condição de ambiente.

\begin{tabular}{|c|c|c|c|c|c|c|c|}
\hline \multirow{2}{*}{ Trat. Térmico } & \multicolumn{7}{|c|}{ DIAS DE ARMAZENAMENTO } \\
\hline & 0 & 1 & 5 & 9 & 13 & 17 & Média \\
\hline $40^{\circ} \mathrm{C}$ & $1,00 \mathrm{~B}$ & $1,00 \mathrm{~B}$ & $2,00 \mathrm{~A}$ & $1,60 \mathrm{AB}$ & $1,60 \mathrm{AB}$ & $1,60 \mathrm{AB}$ & $1,52 \mathrm{a}$ \\
\hline $25^{\circ} \mathrm{C}$ & $1,00 \mathrm{~B}$ & $1,00 \mathrm{~B}$ & $2,00 \mathrm{~A}$ & $1,50 \mathrm{AB}$ & $1,80 \mathrm{~A}$ & $1,80 \mathrm{~A}$ & $1,50 \mathrm{a}$ \\
\hline \multicolumn{8}{|l|}{ Temp. } \\
\hline $25^{\circ} \mathrm{C}$ & $1,00 \mathrm{aB}$ & $1,00 \mathrm{aB}$ & $2,00 \mathrm{aA}$ & $2,00 \mathrm{aA}$ & $2,00 \mathrm{aA}$ & $2,00 \mathrm{aA}$ & 1,66a \\
\hline $14^{\circ} \mathrm{C}$ & $1,00 \mathrm{aB}$ & $1,00 \mathrm{aB}$ & $2,00 \mathrm{aA}$ & $1,70 \mathrm{aA}$ & $1,70 \mathrm{abA}$ & $1,50 \mathrm{bAB}$ & $1,50 \mathrm{ab}$ \\
\hline $8^{\circ} \mathrm{C}$ & $1,00 \mathrm{aB}$ & $1,00 \mathrm{aB}$ & 2,00 aA & $1,00 \mathrm{bB}$ & $1,50 \mathrm{bAB}$ & $1,70 \mathrm{abA}$ & $1,37 \mathrm{~b}$ \\
\hline Média & $1,00 \mathrm{C}$ & $1,00 \mathrm{C}$ & $2,00 \mathrm{~A}$ & $1,58 \mathrm{~B}$ & $1,75 \mathrm{AB}$ & $1,75 \mathrm{AB}$ & - \\
\hline \multicolumn{8}{|c|}{ CV $=17,40 \%$; desvio-padrão= 0,26} \\
\hline
\end{tabular}

\begin{tabular}{|c|c|c|c|c|}
\hline \multirow{2}{*}{ Trat. Térmico } & \multicolumn{4}{|c|}{ DIAS DE ARMAZENAMENTO } \\
\hline & 21 & 25 & 29 & Média \\
\hline $40^{\circ} \mathrm{C}$ & $2,00 \mathrm{~B}$ & $2,00 \mathrm{~B}$ & $2,50 \mathrm{~A}$ & 2,16a \\
\hline $25^{\circ} \mathrm{C}$ & $2,00 \mathrm{~A}$ & $2,00 \mathrm{~A}$ & $2,25 \mathrm{~A}$ & $2,08 a$ \\
\hline \multicolumn{5}{|l|}{ Temperatura } \\
\hline $14^{\circ} \mathrm{C}$ & $2,00 \mathrm{aB}$ & $2,00 \mathrm{aB}$ & 2,75 aA & $2,25 a$ \\
\hline $8^{\circ} \mathrm{C}$ & $2,00 \mathrm{aA}$ & $2,00 \mathrm{aA}$ & $2,00 \mathrm{aA}$ & $2,00 \mathrm{a}$ \\
\hline Média & $2,00 \mathrm{~B}$ & $2,00 \mathrm{~B}$ & $2,37 \mathrm{~A}$ & - \\
\hline
\end{tabular}

Médias seguidas de letras comuns, minúsculas nas colunas e maiúsculas nas linhas, não diferem entre si pelo teste de Tukey $(\mathrm{P}<0,05)$. 
TABELA 2- Luminosidade da polpa de abacaxis 'Pérola', colhidos no estádio de maturação pintado, tratados termicamente a $40^{\circ} \mathrm{C}$ ou não e armazenados por 17 dias, a $25^{\circ} \mathrm{C}$ (ambiente), $14^{\circ} \mathrm{C}$ e $8^{\circ} \mathrm{C}$, quando foram transferidos para condição de ambiente.

\begin{tabular}{|c|c|c|c|c|c|c|c|}
\hline \multirow{2}{*}{ Trat.Térmico } & \multicolumn{7}{|c|}{ DIAS DE ARMAZENAMENTO } \\
\hline & 0 & 1 & 5 & 9 & 13 & 17 & Média \\
\hline $40^{\circ} \mathrm{C}$ & 77,05 & 60,80 & 72,60 & 72,76 & 70,26 & 74,40 & $71,31 \mathrm{a}$ \\
\hline $25^{\circ} \mathrm{C}$ & 77,05 & 72,25 & 70,53 & 71,73 & 70,25 & 72,00 & $72,30 \mathrm{a}$ \\
\hline \multicolumn{8}{|l|}{ Temperatura } \\
\hline $25^{\circ} \mathrm{C}$ & 77,05 & 66,52 & 75,80 & 72,12 & 65,37 & 75,20 & $72,01 \mathrm{a}$ \\
\hline $14^{\circ} \mathrm{C}$ & 77,05 & 66,52 & 69,67 & 70,22 & 72,60 & 76,12 & $72,03 a$ \\
\hline $8^{\circ} \mathrm{C}$ & 77,05 & 66,52 & 69,22 & 74,40 & 72,80 & 68,27 & $71,37 a$ \\
\hline Média & $77,05 \mathrm{~A}$ & $66,52 \mathrm{~B}$ & $71,56 \mathrm{AB}$ & $72,25 \mathrm{AB}$ & $70,25 \mathrm{AB}$ & $73,20 \mathrm{AB}$ & - \\
\hline \multicolumn{8}{|c|}{$C V=9,68 \%$; desvio-padrão $=6,95$} \\
\hline \multirow{2}{*}{ Trat. Térmico } & \multicolumn{7}{|c|}{ DIAS DE ARMAZENAMENTO } \\
\hline & & 21 & 25 & \multicolumn{2}{|c|}{29} & \multicolumn{2}{|c|}{ Média } \\
\hline $40^{\circ} \mathrm{C}$ & \multicolumn{2}{|r|}{77,12} & 67,32 & \multicolumn{2}{|c|}{71,47} & \multicolumn{2}{|c|}{$71,97 a$} \\
\hline $25^{\circ} \mathrm{C}$ & \multicolumn{2}{|c|}{75,47} & 70,05 & \multicolumn{2}{|c|}{76,15} & \multicolumn{2}{|c|}{$73,89 a$} \\
\hline \multicolumn{8}{|l|}{ Temperatura } \\
\hline $14^{\circ} \mathrm{C}$ & \multicolumn{2}{|r|}{78,75} & 67,00 & \multicolumn{2}{|c|}{71,60} & \multicolumn{2}{|c|}{$72,45 a$} \\
\hline $8^{\circ} \mathrm{C}$ & \multicolumn{2}{|c|}{73,85} & 70,37 & \multicolumn{2}{|c|}{76,02} & \multicolumn{2}{|c|}{$73,41 \mathrm{a}$} \\
\hline Média & \multicolumn{2}{|c|}{$76,30 \mathrm{~A}$} & $68,68 \mathrm{~A}$ & \multicolumn{2}{|c|}{$73,81 \mathrm{~A}$} & \multicolumn{2}{|c|}{-} \\
\hline
\end{tabular}

$\mathrm{CV}=9,76 \%$; desvio-padrão= 7,12

Médias seguidas de letras comuns, minúsculas nas colunas e maiúsculas nas linhas, nầo diferem entre si pelo teste de Tukey $(\mathrm{P}<0,05)$.

TABELA 3 - Ângulo hue ou de cor da polpa de abacaxis 'Pérola', colhidos no estádio de maturação pintado, tratados termicamente a $40^{\circ} \mathrm{C}$ ou não e armazenados por 17 dias, a $25^{\circ} \mathrm{C}$ (ambiente), $14^{\circ} \mathrm{C}$ e $8^{\circ} \mathrm{C}$, quando foram transferidos para condição de ambiente.

\begin{tabular}{|c|c|c|c|c|c|c|c|}
\hline \multirow{2}{*}{ Trat.Térmico } & \multicolumn{7}{|c|}{ DIAS DE ARMAZENAMENTO } \\
\hline & 0 & 1 & 5 & 9 & 13 & 17 & Média \\
\hline $40^{\circ} \mathrm{C}$ & 102,5 & 99,1 & 102,6 & 101,9 & 100,5 & 101,8 & $101,4 \mathrm{a}$ \\
\hline $25^{\circ} \mathrm{C}$ & 102,5 & 101,5 & 102,8 & 99,1 & 99,3 & 102,6 & 101,3a \\
\hline \multicolumn{8}{|l|}{ Temperatura } \\
\hline $25^{\circ} \mathrm{C}$ & 102,5 & 100,3 & 102,8 & 101,9 & 99,8 & 101,9 & $101,5 a$ \\
\hline $14^{\circ} \mathrm{C}$ & 102,5 & 100,3 & 102,9 & 99,9 & 99,5 & 102,3 & $100,7 \mathrm{a}$ \\
\hline $8^{\circ} \mathrm{C}$ & 102,5 & 100,3 & 102,2 & 102,6 & 100,4 & 102,3 & $101,7 \mathrm{a}$ \\
\hline Média & $102,5 \mathrm{~A}$ & $100,3 \mathrm{AB}$ & $102,7 \mathrm{~A}$ & $100,5 \mathrm{AB}$ & $99,9 B$ & $102,2 \mathrm{AB}$ & - \\
\hline \multicolumn{8}{|c|}{ CV= 27,84\%; desvio-padrão= 4,48 } \\
\hline \multirow{2}{*}{\multicolumn{2}{|c|}{ Trat. Térmico }} & \multicolumn{6}{|c|}{ DIAS DE ARMAZENAMENTO } \\
\hline & & 21 & \multicolumn{2}{|c|}{\begin{tabular}{|l|l|}
25 & \\
\end{tabular}} & 29 & \multicolumn{2}{|c|}{ Média } \\
\hline $40^{\circ} \mathrm{C}$ & & 103,73 & \multicolumn{2}{|c|}{99,91} & 101,28 & \multicolumn{2}{|c|}{$101,64 a$} \\
\hline $25^{\circ} \mathrm{C}$ & & 102,96 & \multicolumn{2}{|c|}{101,81} & 101,55 & \multicolumn{2}{|c|}{$102,11 \mathrm{a}$} \\
\hline \multicolumn{8}{|l|}{ Temperatura } \\
\hline $14^{\circ} \mathrm{C}$ & & 103,34 & \multicolumn{2}{|c|}{101,80} & 101,36 & \multicolumn{2}{|c|}{$101,58 a$} \\
\hline $8^{\circ} \mathrm{C}$ & & 103,39 & \multicolumn{2}{|c|}{99,92} & 101,48 & \multicolumn{2}{|c|}{$102,16 a$} \\
\hline Média & & $103,34 \mathrm{~A}$ & \multicolumn{2}{|c|}{$100,86 \mathrm{~B}$} & $101,42 \mathrm{~B}$ & \multicolumn{2}{|c|}{-} \\
\hline
\end{tabular}

Médias seguidas de letras comuns, minúsculas nas colunas e maiúsculas nas linhas, não diferem entre sì pelo teste de Tukey $(\mathrm{P}<0,05)$. 
TABELA 4-Cromaticidade da polpa de abacaxis ‘Pérola’, colhidos no estádio de maturação pintado, tratados termicamente a $40^{\circ} \mathrm{C}$ ou não e armazenados por 17 dias, a $25^{\circ} \mathrm{C}$ (ambiente), $14^{\circ} \mathrm{C}$ e $8^{\circ} \mathrm{C}$, quando foram transferidos para condição de ambiente.

\begin{tabular}{|c|c|c|c|c|c|c|c|}
\hline \multirow{2}{*}{ Trat. Térmico } & \multicolumn{7}{|c|}{ DIAS DE ARMAZENAMENTO } \\
\hline & 0 & 1 & 5 & 9 & 13 & 17 & Média \\
\hline $40^{\circ} \mathrm{C}$ & 14,4 & 16,2 & 16,4 & 15,9 & 16,1 & 19,9 & $16,5 a$ \\
\hline $25^{\circ} \mathrm{C}$ & 14,4 & 17,9 & 15,7 & 16,7 & 17,8 & 18,4 & $16,8 \mathrm{a}$ \\
\hline \multicolumn{8}{|l|}{ Temp. } \\
\hline $25^{\circ} \mathrm{C}$ & 14,4 & 17,0 & 15,3 & 15,1 & 16,7 & 20,4 & $16,5 a$ \\
\hline $14^{\circ} \mathrm{C}$ & 14,4 & 17,0 & 16,4 & 17,4 & 17,8 & 19,2 & $17,0 \mathrm{a}$ \\
\hline $8^{\circ} \mathrm{C}$ & 14,4 & 17,0 & 16,5 & 16,5 & 16,3 & 17,9 & $16,4 a$ \\
\hline Média & $14,4 \mathrm{C}$ & $17,0 \mathrm{AB}$ & $16,1 \mathrm{BC}$ & $16,3 \mathrm{BC}$ & $17,0 \mathrm{AB}$ & $19,2 \mathrm{~A}$ & - \\
\hline \multicolumn{8}{|c|}{$C V=12,26 \%$; desvio-padrão $=2,04$} \\
\hline \multirow{2}{*}{\multicolumn{2}{|c|}{ Tratamento Térmico }} & \multicolumn{6}{|c|}{ DIAS DE ARMAZENAMENTO } \\
\hline & & 21 & & 25 & 2 & & Média \\
\hline \multicolumn{2}{|l|}{$40^{\circ} \mathrm{C}$} & 19,86 & \multicolumn{2}{|c|}{19,06} & \multicolumn{2}{|c|}{21,42} & $20,11 a$ \\
\hline \multicolumn{2}{|l|}{$25^{\circ} \mathrm{C}$} & 20,88 & & \multicolumn{2}{|c|}{23,36} & $21,11 \mathrm{a}$ \\
\hline \multicolumn{8}{|l|}{ Temperatura } \\
\hline \multicolumn{2}{|l|}{$14^{\circ} \mathrm{C}$} & 21,00 & \multicolumn{2}{|c|}{19,33} & \multicolumn{2}{|c|}{22,09} & $20,80 \mathrm{a}$ \\
\hline \multicolumn{2}{|l|}{$8^{\circ} \mathrm{C}$} & 19,74 & \multicolumn{2}{|c|}{18,83} & \multicolumn{2}{|c|}{22,69} & $20,42 \mathrm{a}$ \\
\hline \multicolumn{2}{|l|}{ Média } & $20,37 \mathrm{AB}$ & \multicolumn{2}{|c|}{$19,08 \mathrm{~B}$} & \multicolumn{2}{|c|}{$22,39 \mathrm{~A}$} & - \\
\hline
\end{tabular}

$\mathrm{CV}=7,42 \%$; desvio-padrão= 1,53

Médias seguidas de letras comuns, minúsculas nas colunas e maiúsculas nas linhas, não diferem entre si pelo teste de Tukey $(\mathrm{P}<0,05)$.

TABELA 5 - Acidez titulável (AT), expressa em g de ácido cítrico 100 mL-1 de suco de abacaxis 'Pérola', colhidos no estádio de maturação pintado, tratados termicamente a $40^{\circ} \mathrm{C}$ ou não e armazenados por 17 dias, a $25^{\circ} \mathrm{C}$ (ambiente), $14^{\circ} \mathrm{C}$ e $8^{\circ} \mathrm{C}$, quando foram transferidos para condição de ambiente.

\begin{tabular}{|c|c|c|c|c|c|c|c|}
\hline \multirow{2}{*}{ Trat. Térmico } & \multicolumn{7}{|c|}{ DIAS DE ARMAZENAMENTO } \\
\hline & 0 & & 5 & 9 & 13 & 17 & Média \\
\hline $40^{\circ} \mathrm{C}$ & 0,49 & 0,49 & 0,59 & 0,58 & 0,61 & 0,64 & $0,57 b$ \\
\hline $25^{\circ} \mathrm{C}$ & 0,49 & 0,54 & 0,63 & 0,74 & 0,76 & 0,78 & 0,66a \\
\hline \multicolumn{8}{|l|}{ Temp. } \\
\hline $25^{\circ} \mathrm{C}$ & 0,49 & 0,52 & 0,63 & 0,63 & 0,67 & 0,65 & $0,60 \mathrm{a}$ \\
\hline $14^{\circ} \mathrm{C}$ & 0,49 & 0,52 & 0,60 & 0,65 & 0,70 & 0,77 & $0,62 \mathrm{a}$ \\
\hline $8^{\circ} \mathrm{C}$ & 0,49 & 0,52 & 0,60 & 0,70 & 0,70 & 0,70 & $0,62 \mathrm{a}$ \\
\hline Média & $0,49 \mathrm{C}$ & $0,52 \mathrm{BC}$ & $0,61 \mathrm{AB}$ & $0,66 \mathrm{~A}$ & $0,69 \mathrm{~A}$ & $0,71 \mathrm{~A}$ & - \\
\hline \multicolumn{8}{|c|}{$C V=15,68 \%$; desvio-padrão $=0,09$} \\
\hline \multirow{2}{*}{ Trat. Térmico } & \multicolumn{7}{|c|}{ DIAS DE ARMAZENAMENTO } \\
\hline & 21 & & 25 & & 29 & & Média \\
\hline $40^{\circ} \mathrm{C}$ & 0,74 & & 0,64 & & 0,58 & & $0,65 b$ \\
\hline $25^{\circ} \mathrm{C}$ & 0,79 & & 0,79 & & 0,85 & & $0,81 \mathrm{a}$ \\
\hline \multicolumn{8}{|l|}{ Temperatura } \\
\hline $14^{\circ} \mathrm{C}$ & 0,83 & & 0,73 & & 0,74 & & $0,76 a$ \\
\hline $8^{\circ} \mathrm{C}$ & 0,71 & & 0,71 & & 0,68 & & 0,70a \\
\hline Média & $0,77 \mathrm{~A}$ & & $0,72 \mathrm{~A}$ & & $0,71 \mathrm{~A}$ & & - \\
\hline
\end{tabular}

$\mathrm{CV}=13,72 \%$; desvio-padrão= 0,10

Médias seguidas de letras comuns, minúsculas nas colunas e maiúsculas nas linhas, não diferem entre si pelo teste de Tukey $(\mathrm{P}<0,05)$. 
TABELA 6 - Relação entre os teores de SS/AT de suco de abacaxis 'Pérola', colhidos no estádio de maturação pintado, tratados termicamente a $40^{\circ} \mathrm{C}$ ou não e armazenados por 17 dias, a $25^{\circ} \mathrm{C}$ (ambiente), $14^{\circ} \mathrm{C}$ e $8^{\circ} \mathrm{C}$, quando foram transferidos para condição de ambiente.

\begin{tabular}{|c|c|c|c|c|c|c|c|}
\hline \multirow{2}{*}{ Trat. Térmico } & \multicolumn{7}{|c|}{ DIAS DE ARMAZENAMENTO } \\
\hline & 0 & 1 & 5 & 9 & 13 & 17 & Média \\
\hline $40^{\circ} \mathrm{C}$ & 27,7 & 25,0 & 22,1 & 23,8 & 22,4 & 21,2 & $23,7 a$ \\
\hline $25^{\circ} \mathrm{C}$ & 27,7 & 23,6 & 20,3 & 17,7 & 17,5 & 17,4 & $20,7 b$ \\
\hline \multicolumn{8}{|l|}{ Temp. } \\
\hline $25^{\circ} \mathrm{C}$ & 27,7 & 24,3 & 20,3 & 21,5 & 20,3 & 21,4 & $22,6 a$ \\
\hline $14^{\circ} \mathrm{C}$ & 27,7 & 24,3 & 22,2 & 20,5 & 19,6 & 18,1 & $22,0 \mathrm{a}$ \\
\hline $8^{\circ} \mathrm{C}$ & 27,7 & 24,3 & 21,1 & 20,2 & 19,9 & 18,3 & $21,9 a$ \\
\hline Média & $27,7 \mathrm{~A}$ & $24,3 \mathrm{AB}$ & $21,2 \mathrm{BC}$ & $20,7 \mathrm{BC}$ & $19,9 \mathrm{BC}$ & $19,3 \mathrm{C}$ & - \\
\hline \multicolumn{8}{|c|}{$C V=18,14 \%$; desvio-padrão $=4,03$} \\
\hline \multirow{2}{*}{ Trat. Térmico } & \multicolumn{7}{|c|}{ DIAS DE ARMAZENAMENTO } \\
\hline & \multicolumn{2}{|c|}{21} & 25 & \multicolumn{2}{|c|}{29} & \multicolumn{2}{|r|}{ Média } \\
\hline $40^{\circ} \mathrm{C}$ & \multicolumn{2}{|c|}{17,8} & 21,2 & \multicolumn{3}{|c|}{21,2} & $21,0 a$ \\
\hline $25^{\circ} \mathrm{C}$ & \multicolumn{2}{|c|}{17,4} & 17,1 & \multicolumn{3}{|c|}{17,1} & $17,1 b$ \\
\hline \multicolumn{8}{|l|}{ Temperatura } \\
\hline $14^{\circ} \mathrm{C}$ & \multicolumn{2}{|c|}{15,7} & 19,7 & \multicolumn{3}{|c|}{19,2} & $18,2 \mathrm{a}$ \\
\hline $8^{\circ} \mathrm{C}$ & \multicolumn{2}{|c|}{19,6} & 18,6 & \multicolumn{2}{|r|}{21,8} & \multicolumn{2}{|r|}{$20,0 \mathrm{a}$} \\
\hline Média & \multicolumn{2}{|c|}{$17,6 \mathrm{~A}$} & $19,1 \mathrm{~A}$ & \multicolumn{3}{|c|}{$20,5 \mathrm{~A}$} & - \\
\hline
\end{tabular}

$\mathrm{CV}=20,77 \%$; desvio-padrão= 3,97

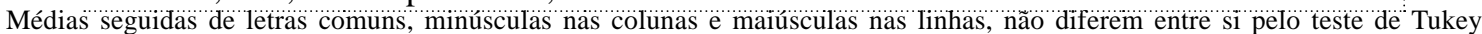
$(\mathrm{P}<0,05)$.

TABELA 7 - Teor de açúcares solúveis totais (g glicose $100 \mathrm{~g}^{-1}$ ) no suco de abacaxis 'Pérola', colhidos no estádio de maturação pintado, tratados termicamente a $40^{\circ} \mathrm{C}$ ou não e armazenados por 17 dias, a $25^{\circ} \mathrm{C}$ (ambiente), $14^{\circ} \mathrm{C}$ e $8^{\circ} \mathrm{C}$, quando foram transferidos para condição de ambiente.

\begin{tabular}{|c|c|c|c|c|c|c|c|}
\hline \multirow{2}{*}{ Trat. Térmico } & \multicolumn{7}{|c|}{ DIAS DE ARMAZENAMENTO } \\
\hline & 0 & 1 & 5 & 9 & 13 & 17 & Média \\
\hline $40^{\circ} \mathrm{C}$ & 13,40 & 8,3 & 11,21 & 10,25 & 10,55 & 12,18 & $10,98 a$ \\
\hline $25^{\circ} \mathrm{C}$ & 13,40 & 8,25 & 10,48 & 9,28 & 9,83 & 12,18 & $10,57 \mathrm{a}$ \\
\hline \multicolumn{8}{|l|}{ Temp. } \\
\hline $25^{\circ} \mathrm{C}$ & 13,40 & 8,27 & 10,45 & 10,07 & 9,62 & 11,85 & $10,61 a$ \\
\hline $14^{\circ} \mathrm{C}$ & 13,40 & 8,27 & 12,02 & 10,4 & 10,67 & 11,95 & $11,12 \mathrm{a}$ \\
\hline $8^{\circ} \mathrm{C}$ & 13,40 & 8,27 & 10,07 & 8,82 & 10,27 & 12,75 & $10,60 \mathrm{a}$ \\
\hline Média & $13,40 \mathrm{~A}$ & $8,27 \mathrm{D}$ & $10,85 B C$ & $9,76 \mathrm{C}$ & $10,19 \mathrm{C}$ & $12,18 \mathrm{AB}$ & - \\
\hline \multicolumn{8}{|c|}{$\mathrm{CV}=10,13 \%$; desvio-padrão $=1,09$} \\
\hline
\end{tabular}

\begin{tabular}{l|cc|c|c|}
\hline \multirow{2}{*}{ Trat. Térmico } & \multicolumn{4}{|c|}{ DIAS DE ARMAZENAMENTO } \\
\cline { 2 - 6 } & 21 & 25 & 29 & Média \\
\hline $40^{\circ} \mathrm{C}$ & 12,35 & 11,76 & 11,66 & $11,92 \mathrm{~b}$ \\
$25^{\circ} \mathrm{C}$ & 13,53 & 15,37 & 14,53 & $14,47 \mathrm{a}$ \\
\hline Temperatura & \multicolumn{5}{c}{} \\
\hline $14^{\circ} \mathrm{C}$ & 12,46 & 14,61 & 13,41 & $12,96 \mathrm{a}$ \\
$8^{\circ} \mathrm{C}$ & 13,41 & 12,51 & 14,40 & $13,44 \mathrm{a}$ \\
\hline Média & $12,94 \mathrm{~A}$ & $13,56 \mathrm{~A}$ & $13,10 \mathrm{~A}$ & - \\
\hline
\end{tabular}

$\mathrm{CV}=17,45 \%$; desvio-padrão= 2,30

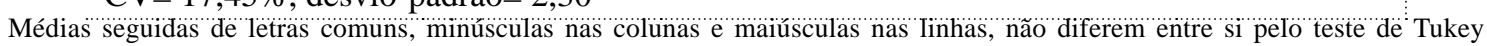
$(\mathrm{P}<0,05)$. 
TABELA 8 - Teores de açúcares redutores (g glicose $100 \mathrm{~g}^{-1}$ ) no suco de abacaxis ‘Pérola’, colhidos no estádio de maturação pintado, tratados termicamente a $40^{\circ} \mathrm{C}$ ou não e armazenados por 17 dias, a $25^{\circ} \mathrm{C}$ (ambiente), $14^{\circ} \mathrm{C}$ e $8^{\circ} \mathrm{C}$, quando foram transferidos para condição de ambiente.

\begin{tabular}{|c|c|c|c|c|c|c|c|}
\hline \multirow{2}{*}{ Trat. Térmico } & \multicolumn{7}{|c|}{ DIAS DE ARMAZENAMENTO } \\
\hline & 0 & 1 & 5 & 9 & 13 & 17 & Média \\
\hline $40^{\circ} \mathrm{C}$ & 2,39 & 2,24 & 2,28 & 2,12 & 2,11 & 1,10 & $2,22 \mathrm{a}$ \\
\hline $25^{\circ} \mathrm{C}$ & 2,39 & 2,09 & 2,49 & 2,14 & 2,51 & 2,25 & $2,31 \mathrm{a}$ \\
\hline \multicolumn{8}{|l|}{ Temp. } \\
\hline $25^{\circ} \mathrm{C}$ & 2,39 & 2,17 & 2,37 & 2,05 & 2,30 & 2,04 & $2,22 \mathrm{a}$ \\
\hline $14^{\circ} \mathrm{C}$ & 2,39 & 2,17 & 2,42 & 2,28 & 2,23 & 1,99 & $2,24 a$ \\
\hline $8^{\circ} \mathrm{C}$ & 2,39 & 2,17 & 2,38 & 2,07 & 2,39 & 2,64 & $2,34 a$ \\
\hline Média & $2,39 \mathrm{~A}$ & $2,17 \mathrm{~A}$ & $2,39 \mathrm{~A}$ & $2,13 \mathrm{~A}$ & $2,31 \mathrm{~A}$ & $2,22 \mathrm{~A}$ & - \\
\hline \multicolumn{8}{|c|}{$\mathrm{CV}=15,90 \%$; desvio-padrão $=0,36$} \\
\hline \multirow{2}{*}{ Trat. Térmico } & \multicolumn{7}{|c|}{ DIAS DE ARMAZENAMENTO } \\
\hline & \multicolumn{2}{|c|}{21} & \multicolumn{2}{|c|}{\begin{tabular}{l|l}
25 & \\
\end{tabular}} & 29 & \multicolumn{2}{|r|}{ Média } \\
\hline $40^{\circ} \mathrm{C}$ & \multicolumn{2}{|c|}{2,31} & \multicolumn{2}{|l|}{2,75} & 2,70 & \multicolumn{2}{|r|}{$2,59 a$} \\
\hline $25^{\circ} \mathrm{C}$ & \multicolumn{2}{|c|}{2,41} & \multicolumn{2}{|l|}{2,62} & 2,89 & \multicolumn{2}{|r|}{$2,64 a$} \\
\hline \multicolumn{8}{|l|}{ Temperatura } \\
\hline $14^{\circ} \mathrm{C}$ & \multicolumn{2}{|c|}{2,20} & \multicolumn{2}{|l|}{2,54} & 2,42 & \multicolumn{2}{|r|}{$2,39 b$} \\
\hline $8^{\circ} \mathrm{C}$ & \multicolumn{2}{|c|}{2,51} & \multicolumn{2}{|l|}{2,83} & 3,16 & \multicolumn{2}{|r|}{$2,84 a$} \\
\hline Média & \multicolumn{2}{|c|}{$2,36 \mathrm{~A}$} & \multicolumn{2}{|l|}{$2,69 \mathrm{~A}$} & $2,79 \mathrm{~A}$ & \multicolumn{2}{|r|}{ - } \\
\hline
\end{tabular}

CV=16,21 \%; desvio-padrão= 0,42

Médiaas seguidas de letras comuns, minúsculäs nas colunas e maiüsculäs nas linhaas, não diferem entre si pelo teste de Tukey $(\mathrm{P}<0,05)$

TABELA 9 - Teor de ácido ascórbico (mg $100 \mathrm{~g}^{-1}$ ) no suco de abacaxis 'Pérola', colhidos no estádio de maturação pintado, tratados termicamente a $40^{\circ} \mathrm{C}$ ou não e armazenados por 17 dias, a $25^{\circ} \mathrm{C}$ (ambiente), $14^{\circ} \mathrm{C}$ e $8^{\circ} \mathrm{C}$, quando foram transferidos para condição de ambiente.

\begin{tabular}{|c|c|c|c|c|c|c|c|}
\hline \multirow{2}{*}{ Trat. Térmico } & \multicolumn{7}{|c|}{ DIAS DE ARMAZENAMENTO } \\
\hline & 0 & & 5 & 9 & 13 & 17 & Média \\
\hline $40^{\circ} \mathrm{C}$ & 10,55 & 12,70 & 20,48 & 16,15 & 19,55 & 16,03 & $15,91 \mathrm{a}$ \\
\hline $25^{\circ} \mathrm{C}$ & 10,55 & 14,50 & 19,31 & 18,60 & 19,23 & 15,76 & $16,32 \mathrm{a}$ \\
\hline \multicolumn{8}{|l|}{ Temp. } \\
\hline $25^{\circ} \mathrm{C}$ & 10,55 & 13,60 & 20,85 & 18,87 & 22,30 & 16,01 & $17,03 a$ \\
\hline $14^{\circ} \mathrm{C}$ & 10,55 & 13,60 & 17,32 & 17,4 & 17,82 & 17,25 & $15,65 a$ \\
\hline $8^{\circ} \mathrm{C}$ & 10,55 & 13,60 & 21,52 & 15,85 & 18,05 & 14,42 & $15,66 \mathrm{a}$ \\
\hline Média & $10,55 \mathrm{C}$ & $13,60 \mathrm{BC}$ & $19,90 \mathrm{~A}$ & $17,35 \mathrm{AB}$ & $19,39 \mathrm{~A}$ & $15,90 \mathrm{ABC}$ & - \\
\hline \multicolumn{8}{|c|}{$\mathrm{CV}=27,84 \%$; desvio-padrão $=4,48$} \\
\hline \multirow{2}{*}{ Trat. Térmico } & \multicolumn{7}{|c|}{ DIAS DE ARMAZENAMENTO } \\
\hline & & & \multirow{2}{*}{\multicolumn{2}{|c|}{25}} & 29 & \multicolumn{2}{|c|}{ Média } \\
\hline $40^{\circ} \mathrm{C}$ & \multicolumn{2}{|r|}{16,19} & & & 21,38 & \multicolumn{2}{|c|}{$18,11 a$} \\
\hline $25^{\circ} \mathrm{C}$ & \multicolumn{2}{|r|}{15,85} & \multicolumn{2}{|c|}{$\begin{array}{l}27,50 \\
18,48\end{array}$} & 21,94 & \multicolumn{2}{|c|}{$18,75 a$} \\
\hline \multicolumn{8}{|l|}{ Temperatura } \\
\hline $14^{\circ} \mathrm{C}$ & \multicolumn{2}{|r|}{18,26} & \multicolumn{2}{|c|}{18,94} & 22,63 & \multicolumn{2}{|c|}{$19,94 a$} \\
\hline $8^{\circ} \mathrm{C}$ & \multicolumn{2}{|r|}{13,77} & \multicolumn{2}{|c|}{16,31} & 20,69 & \multicolumn{2}{|c|}{$16,92 \mathrm{a}$} \\
\hline Média & \multicolumn{2}{|c|}{$16,02 \mathrm{~A}$} & \multicolumn{2}{|c|}{$17,62 \mathrm{~A}$} & $21,66 \mathrm{~A}$ & \multicolumn{2}{|c|}{-} \\
\hline
\end{tabular}

CV= 23,49\%; desvio-padrão= 4,33

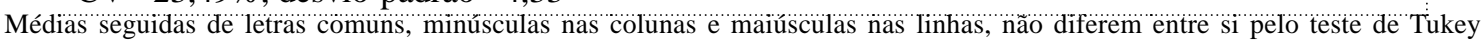
$(\mathrm{P}<0,05)$. 


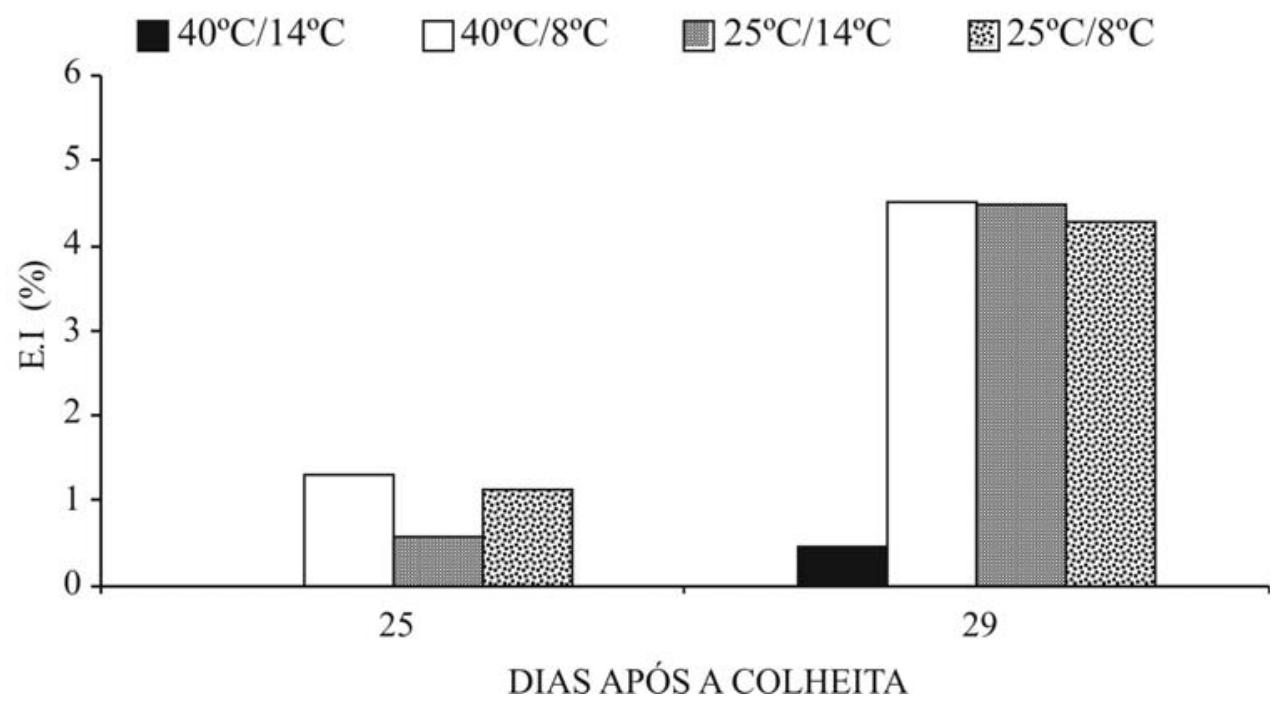

FIGURA 1- Índice de escurecimento interno (EI) em frutos de abacaxis 'Pérola', colhidos no estádio de maturação pintado, tratados termicamente a $40^{\circ} \mathrm{C}$ ou não e armazenados por 17 dias, a $25^{\circ} \mathrm{C}$ (ambiente), $14^{\circ} \mathrm{C}$ e $8^{\circ} \mathrm{C}$, quando foram transferidos para condição de ambiente.

\section{CONCLUSÕES}

Os frutos mantidos sob refrigeração $\left(8^{\circ} \mathrm{C} \mathrm{e}\right.$ $14^{\circ} \mathrm{C}$ ) apresentaram maior vida útil do que os mantidos em condição ambiente $\left(25^{\circ} \mathrm{C}\right)$, sendo descartados após 17 dias de armazenamento. O tratamento térmico a $40^{\circ} \mathrm{C}$, por 24 horas, reduziu a intensidade do escurecimento interno nos frutos sem influir na qualidade e na conservação dos mesmos

\section{REFERÊNCIAS}

AKAMINE, E. K. Postharvest control of endogenous brown spot in fresh Australian roth heat. HortScience, St Joseph, v. 11, n. 6, p. 586-588, 1976.

AOAC. Official methods of analysis of the Association of Official Analytical Chemists. 16. ed. Washington: AOAC, 1997. v. 2, p. 37-10, 42-42, 44-43, 45-16.

BLEINROTH, E. W. Matéria-Prima. In: ITAL. Abacaxi: cultura, matéria-prima, processamento e aspectos econômicos. 2. ed. Campinas: ITAL, 1987. p. 133-64. (Série Frutas Tropicais, 2).
BOTREL, N. Efeito do peso do fruto no escurecimento interno e qualidade do abacaxi 'Smooth Cayenne’. 1991. 81f. Dissertação (Mestrado em Fitotecnia) - Escola Superior de Agricultura de Lavras, Lavras, 1991.

CARVALHO, V. D. de. Composição, colheita, embalagem e transporte do fruto. In: CUNHA, G. A P.; CABRAL, J. R. S.; SOUZA, L. F. S. O abacaxizeiro: cultivo, agroindústria e economia. Brasília: Embrapa, 1999. p. 367-388.

CEAGESP. Programa brasileiro para a modernização da horticultura: normas de classificação do abacaxi. São Paulo: Centro de Qualidade em Horticultura, 2000. (Documentos, 24).

CHITARRA, M. I.; CHITARRA, A. B. Pós-colheita de frutos e hortaliças: fisiologia e manuseio. 2. ed. Lavras: UFLA, 2005. 783p.

DUBOIS, M.; GILLES, K. A.; HAMILTON, J. K.; REBER, P. A.; SMITH, F. Calorimetric method for determination of sugar and related substances. Analytical Chemistry, Washington, v. 2, n. 3, p. 350-356, 1956. 
DULL, G. G. The pineapple: general. In: HULME, A. C. (Ed.). The biochemistry of fruits and their products. London: Academic Press, 1971. v. 2, p. 303-323.

GARCIA, E. E. C.; GARCIA, A. E.; ARDITO, E. F.; BORDIN, M. R. Embalagem. In: GORGATTI NETTO, A. et al. Abacaxi para exportação: procedimentos de colheita e pós- colheita. Brasília: EMBRAPA, 1996. p.32-41. (Série Publicações Técnicas FRUPEX, 23).

GIACOMELLI, E. J. Expansão da abacaxicultura no Brasil. Campinas: Fundação Cargill, 1982. 79p.

GONÇALVES, N. B. Efeito da aplicação de cloreto de cálcio associado ao tratamento hidrotérmico sobre a composição química e suscetibilidade ao escurecimento interno do abacaxi cv. Smooth Cayenne. 1998. 96f. Tese (Doutorado em Fitotecnia)- Universidade Federal de Lavras, Lavras, 1998.

GONÇALVES, N. B.; CARVALHO, V. D. de. Características da fruta. In: GONÇALVES, N. B. (Org.). Abacaxi pós-colheita. Brasília: Ministério da Agricultura e do Abastecimento; Embrapa. Comunicação para Transferência de Tecnologia. 2000. cap. 2, p. 13-27 (Frutas do Brasil, 5)

GORGATTI NETTO, A.; CARVALHO, V. D. de; BOTREL, N.; BLEINROTH, E. W.; MATALLO, M.; GARCIA, A. E.; ARDITO, E. F. G.; GARCIA, E. E. R.; BORDIN, M. R. Abacaxi para exportação: procedimentos de colheita e pós-colheita. Brasília: EMBRAPA-SPI, 1996. 41p. (Série Publicações Técnicas FRUPEX, 23).

MILLER, G. L. Use of dinitrosalicylic acid reagent for determination of reducing sugars. Analytical Chemistry, Washington, v. 31, n. 3, p. 426-428, 1959.

SELVARAJAH, S.; HERATH, H.M.W. Effect of an edible coating on some quality and physico-chemical parameters of pineapple during cold storage: Tropical Agricultural Research, Amsterdan, v. 18, p. 77-89, 1997.

SILVA, J. M. da. Uso de atmosfera modificada no armazenamento do abacaxi (Ananas comosus $\mathbf{L}$. Merr) cv. Smooth Cayenne. 1997. 85 f. Dissertação (Mestrado em Fisiologia Vegetal) - Universidade Federal de Lavras, Lavras, 1997.
SILVA, M. A. Fisiologia pós-colheita de abacaxi cvs. Pérola e Smooth Cayenne. 1980. 203 f. Dissertação (Mestrado em Engenharia Agrícola) - Faculdade de Engenharia Agrícola, Universidade de Campinas, Campinas, 1980.

SOUTO, R. F.; DURIGAN J. F.; SOUZA, B. S. de; DONADON, J. R.; MENEGUCCI, J. L P. Conservação pós-colheita de abacaxi 'Pérola' colhido no estádio de maturação "pintado" associando-se refrigeração e atmosfera modificada. Revista Brasileira de Fruticultura, Jaboticabal, v. 26, n.1, p. 24-28, 2004.

STROHECKER, R.L.; HENNING, H.M. Análises de vitaminas: métodos comprobados. Madrid: Paz Montalvo, 1967. 428p.

TANG-YOULIN; ZHOU-YUCHAN; TAN-XING, J. I. E.; TANG-Y, L.; ZHOU-Y,C.; TAN-X, J.; MARTIN-PREVEL, P.; HUGON-R. (Ed.). A study on factor inducing and controlling postharvest blackheart in pineapples. Acta Horticulturae, Wageningen, v. 9, n. 425, p. 595-603, 1997.

THÉ, P. M. P.; BOLTREL, N.; NUNES, R. de P.; CARVALHO, V. D. de. Influência de tratamentos pós-colheita sobre a atividade enzimática de abacaxi cv. Smooth Cayenne. Boletin CEPPA, Curitiba, v. 24, n.2, p.423-442, 2006.

THÉ, P. M. P.; CARAVALHO, V. D. de ; ABREU, C. M. P. de.; NUNES, R. de P.; PINTO, N. A V. D. Efeito da temperatura de armazenamento e do estádio de maturação sobre a composição química do abacaxi cv. Smooth Cayenne L. Ciências e Agrotécnologia, Lavras, v. 25, n. 2, p. 356-363, 2001.

THÉ, P. M. P.; GONÇALVES, B. N.; NUNES, R. de P.; MORAIS, A. R. de; PINTO, N. A. V. D.; FERNANDES, S. M.; CARVALHO, V. D. Efeitos de tratamentos pós-colheita sobre fatores relacionados à qualidade de abacaxi cv. Smooth Cayenne. Revista Brasileira Agrociência, Pelotas, v. 9, n.2, p.163-170, 2003.

WOLF, A.B.; MACRAE, E.A.; SPOONER, K.J.; REDEWELL, R.J. Changes to physical properties of the cell wall and polyuronides in response to heat treatment of 'Fuyu' persimmon that alleviate chilling injury. Journal of the American Society for Horticultural Science, Alexandria, v. 122, p. 698-702, 1997. 